\title{
Endometrial cancer combined with polycystic ovary syndrome in 9 women under 40-years old: A case report
}

\author{
XIBIAO JIA, LINGYUN YANG, PAN XU, NINGWEI LI, CEN CHEN and HONGJING WANG \\ Key Laboratory of Birth Defects and Related Diseases of Women and Children, Ministry of Education, \\ West China Second University Hospital, Sichuan University, Chengdu, Sichuan 610041, P.R. China
}

Received February 20, 2020; Accepted July 23, 2020

DOI: 10.3892/br.2020.1357

\begin{abstract}
The aim of the present study was to discuss the clinicopathological characteristics of endometrial carcinoma in young females with polycystic ovary syndrome (PCOS), and to review the current literature. A total of 9 patients with histopathologically confirmed endometrial cancer in young females with PCOS at the West China Second Hospital of Sichuan University between December 2007 and September 2013 were included. The clinicopathological characteristics of the patients were analyzed. The age range of the patients was 24-38 years (median age, 29), all of the cases had abnormal vaginal bleeding and endometrioid adenocarcinoma was the most common histopathological subtype observed (8 cases, $88.9 \%$ ). Of the patients, 2 had well-differentiated cases and 7 patients had moderately differentiated cases. None of the patients received regular PCOS treatments and did not turn up for regular check-ups before they were diagnosed with endometrial carcinoma. Additionally, 7 patients received staging laparotomy with a total abdominal hysterectomy and bilateral salpingo-oophorectomy, 1 patient underwent an endometrial resection under hysteroscopy and the final patient received a high-dose of medroxyprogesterone treatment without surgery. In conclusion, doctors should take into consideration that young women with PCOS may also exhibit an endometrial carcinoma, and diagnosing and treating the endometrial carcinoma as early as possible in the young patients with PCOS is necessary.
\end{abstract}

\section{Introduction}

Polycystic ovary syndrome (PCOS) is a heterogeneous disorder affecting $8-17 \%$ of women of reproductive-age,

Correspondence to: Dr Hongjing Wang, Key Laboratory of Birth Defects and Related Diseases of Women and Children, Ministry of Education, West China Second University Hospital, Sichuan University, 20 People South Road Section 3, Chengdu, Sichuan 610041, P.R. China

E-mail: pubgchinawest@126.com

Key words: polycystic ovary syndrome, endometrial cancer, young age and it is the most common endocrinopathy in women (1). The characteristics of PCOS include oligo- or anovulation, hypertestosteronism, obesity and type II diabetes (2).

Endometrial carcinoma is the most common gynecological malignancy, and the disease is most commonly observed in elder women (3). The median age of the patients with endometrial carcinoma was 61 years with $75-80 \%$ being post-menopausal, and only $3-5 \%$ of patients were $<40$ years old (4). Most patients with endometrial carcinoma are postmenopausal patients; however, $4-14 \%$ of cases are observed in younger patients $(<45$ years of age) $(5,6)$. An increasing number of clinical trials and studies are focused on the correlation between PCOS and endometrial cancer, as the major characteristics of PCOS are also risk factors of endometrial carcinoma (7). However, the mechanisms which are shared between the two conditions have not been extensively studied, and most studies are performed based on the assumption that chronic anovulation is a major factor in both conditions, resulting in high estrogen levels which affects the endometrium. Park et al (8) reported that a thick end endometrium $(\sim 8.5 \mathrm{~mm})$ was a risk factor of developing endometrial hyperplasia and endometrial cancer in patients with PCOS. Additionally, it has been shown that polycystic ovarian morphology is significantly more common in younger patients (20-40 years old) with endometrial carcinoma (9). The present study analyzed the characteristics of patients with PCOS and endometrial adenocarcinoma who visited the West China Second Hospital of Sichuan University, and a review of the current literature is performed as well.

\section{Patients and methods}

The present study was performed at the Key Laboratory of Birth Defects and Related Diseases of Women and Children, Ministry of Education of West China Second University Hospital, Sichuan University (Chengdu, China). All of the patients had histopathologically confirmed endometrial carcinoma between December 2007 and September 2013. The clinicopathological characteristics of the patients were analyzed, including their age, presenting symptoms, body mass index, parity, International Federation of Gynecology and Obstetrics (FIGO) staging (10), histology, tumor grade, cervical involvement, depth of myometrial invasion, lymph node involvement, treatment and adjuvant therapy. The age range of the patients was $24-38$ years and the median age was 29 years. 
Postoperative adjuvant therapies were based on histopathological findings. Diagnosis of PCOS was performed in accordance with the American Society of Reproductive Medicine/European Society for Human Reproduction and Embryology diagnostic criteria (11). A necessary criterion was polycystic ovaries, which were detected using ultrasound or the pathological examination of surgical specimens. During the study period, all of the patients underwent a regular physical examination, cytological smear, serum tumor marker measurements, B-type ultrasonic inspection, and abdominal computed tomography or positron emission tomography-computed tomography.

\section{Results}

Of the total 229 patients with endometrial cancer aged $\leq 40$ years treated at the West China Second University Hospital between December 2007 and September 2013, only 9 patients also presented with PCOS. Table I summarizes the demographic data and Table II summarizes the clinicopathological data. Of the 9 patients, 8 patients were women who were nulliparous and 1 patient had one child. All patients complained of abnormal vaginal bleeding. The endometrial thickness of the cases ranged from 3-13.5 mm and the median thickness was $6.1 \mathrm{~mm}$. Additionally, 6 patients (66.7\%) were classed as obese (body mass index $>25 \mathrm{~kg} / \mathrm{m}^{2}$ ). Endometrioid adenocarcinoma was the most common histopathological subtype of endometrial cancer observed (8 cases, $88.9 \%$ ) and 1 patient had adenocarcinoma with squamous differentiation. The FIGO stage of every patient was stage I; 2 patients had well-differentiated cancer and 7 patients had moderately differentiated cancer. In 5 patients $(55.6 \%), \leq 50 \%$ depth myometrial invasion was observed and the other 4 cases did not show evidence of myometrial invasion; 1 patient (11.1\%) had cervical involvement and 7 cases (58.8\%) had undergone lymph node dissections, but none of these patients exhibited signs of lymph node involvement. Additionally, 7 patients underwent staging laparotomies with total abdominal hysterectomies and bilateral salpingo-oophorectomies, 1 patient underwent endometrial resection under hysteroscopy and the other patient received a high-dose of medroxyprogesterone treatment without surgery. All 9 patients were followed up for 38 months, and none of them succumbed to the disease during the follow up period. Throughout the study period, 2 patients received postoperative chemotherapy. The chemotherapeutic regimen was paclitaxel and platinum, which consisted of paclitaxel $\left(135-175 \mathrm{mg} / \mathrm{m}^{2}\right.$ in $500 \mathrm{ml}$ saline, infused intravenously continuously on day 1) and platinum $\left(60 \mathrm{mg} / \mathrm{m}^{2}\right.$ dissolved in $500 \mathrm{ml}$ saline and infused over $4 \mathrm{~h}$ on day 2). This regimen was repeated every 4 weeks for 3 cycles of endometrial thickness. At the time of writing, 7 cases (77.8\%) were alive and free of the disease following treatment, while treatment failed in 2 cases during the follow-up period.

\section{Discussion}

Endometrial cancer is a malignancy arising from the uterine endometrium or lining. While endometrial cancer is rarely observed in younger women, studies have shown the prognostic outcomes of younger women with endometrial
Table I. Clinicopathological characteristics of the patients.

\begin{tabular}{lc}
\hline Variable & $\mathrm{n}(\%)$ \\
\hline Age, years & \\
Median & 29 \\
Range & $24-38$ \\
$\quad$ Standard deviation & 5.02 \\
Presenting symptoms & \\
$\quad$ Abnormal vaginal bleeding & $9(100)$ \\
Endometrial thickness, mm & \\
$\quad$ Median & 6.1 \\
Range & $3-13.5$ \\
Body mass index & \\
$\geq 25$ & $6(66.7)$ \\
$<25$ & \\
Parity & \\
0 & $8(88.9)$ \\
1 & $1(11.1)$ \\
\hline
\end{tabular}

Table II. Histopathological characteristics of the patients.

\begin{tabular}{lc}
\hline Histology & $\mathrm{n}(\%)$ \\
\hline Endometrioid adenocarcinoma & $8(88.9)$ \\
Adenocarcinoma with squamous differentiation & $1(11.1)$ \\
FIGO stage & \\
I & $8(88.9)$ \\
II & $1(11.1)$ \\
Grade & \\
1 & $2(22.2)$ \\
2 & $7(77.8)$ \\
Cervical involvement & \\
No & $8(88.9)$ \\
Yes & $1(11.1)$ \\
Depth of myometrial invasion & \\
$\leq 50 \%$ & $5(55.6)$ \\
None & $4(44.4)$ \\
Lymph node involvement & \\
No & $9(100)$ \\
Yes & $0(0)$ \\
\hline
\end{tabular}

FIGO, International Federation of Gynecology and Obstetrics.

carcinoma are less favorable than those of older women (12-14). Laughlin-Tommaso et al (10) reported a possible discordance between myometrial invasion, tumor grade and lymph node involvement in younger women. However, Hanprasertpong et al (15) found that women with endometrial cancer aged $<45$ years were most commonly diagnosed with early stage and well differentiated cancer. As the number of young women with endometrial cancer is increasing (12), and the majority of women younger women have not given birth, 
the treatment of endometrial cancer in younger women is increasingly challenging (16).

Previous studies have reported that nulliparity (resulting from infertility due to chronic anovulation), obesity and chronic anovulation occurs in younger patients with endometrial carcinoma (16). These characteristics are also typical features observed in patients with PCOS, and PCOS is the most common cause of anovulation and nulliparity in young women. Cheung (17) reported that the rate of endometrial hyperplasia in PCOS without regular treatment was $35.7 \%$, and another study showed that the prevalence of endometrial malignancy in patients with polycystic ovaries was $37 \%$ (14).

Therefore, a possible association between PCOS and endometrial cancer was considered a possibility. An association between PCOS and endometrial carcinoma was first suggested in 1949 (18). Types of cancer associated with chronic hormone stimulation in women include endometrial, ovarian and breast cancer. In women with PCOS, chronic estrogen stimulation often causes endometrial hyperplasia or endometrial cancer $(7,19-21)$. In the present study, the patients recruited were relatively young. Thus, it is possible that genetic factors may be strongly associated with the development of endometrial cancer rather than or in addition to PCOS.

PCOS is characterized by chronic anovulation, nulliparity and high estrogen level exposure, which have also been shown to be associated with the development of endometrial hyperplasia leading to endometrial cancer. A related study showed that the endometrium of patients with PCOS showed a higher concentration of androgen and estrogen receptors, and this may underlie the development of endometrial hyperplasia and endometrial carcinoma (22). In the patients recruited in another study, 8 patients $(88.9 \%)$ were nulliparous and all of them had abnormal vaginal bleeding. Studies have suggested that patients should be evaluated via endometrial biopsy when they exhibited the following factors: Endometrial stripe thickness $>7 \mathrm{~mm}$ or a menstrual cycle for $>3$ months, endometrial stripe thickness $>12 \mathrm{~mm}$ and long-term treatment with exogenous estrogens $(8,17)$.

Another characteristic of PCOS is insulin resistance, which increases the risk of developing type 2 diabetes mellitus, a condition that results in elevated fasting glucose levels (19). Additionally, alterations in the glucose levels may be used to predict development of endometrial cancer, as cancer cells require an increased demand for glucose and thrive in hyperglycemic environments (23). Obesity has also been found to be strongly associated with the risk of endometrial cancer risk in patients with PCOS. Furberg and Thune (24) found that obesity increases the risk of endometrial cancer 2-fold compared with non-obese patients. The results of the present study showed that six cases $(66.7 \%)$ were obese, supporting the previous study.

Previous studies have examined the association between PCOS and endometrial carcinoma at the molecular level. Overexpression of osteopontin, an oncogene involved in carcinogenesis, is observed in the endometrium of patients with PCOS (25). It has also been shown that Cyr61 protein expression levels are increased in patients with PCOS with endometrial cancer (26).

A previous study showed that patients with PCOS presented with well-differentiated or moderately-differentiated instances of endometrial cancer, and the patients were often diagnosed at an early stage with a low risk of metastasis (27). In the present study, 2 patients had well-differentiated instances and 7 patients had moderately-differentiated instances of endometrial cancer. All of the patients were stage I and none of them had lymph node involvement.

The standard treatment for patients with endometrial cancer is a hysterectomy and a bilateral salpingo-oophorectomy (3). In young women who present with a low histological grade and early-stage disease, conservative hormonal therapy may be used with close follow-up (28). In the patients recruited for the present study, 7 patients underwent a staging laparotomy with a total abdominal hysterectomy and a bilateral salpingo-oophorectomy, 1 patient underwent endometrial resection under hysteroscopy and the other patient received a high-dose of medroxyprogesterone treatment without surgery.

The present study highlights the risk of developing endometrial cancer in women with PCOS. In young women who complain about abnormal vaginal bleeding and nulliparity, a diagnosis of PCOS should be considered. After confirmatory diagnosis of PCOS, regular treatment and follow-up are important. A staging laparotomy with a total abdominal hysterectomy and a bilateral salpingo-oophorectomy is the standard treatment. Long-term progestogen treatment should be used in patients who present with a low histological grade and early-stage disease.

\section{Acknowledgements}

The author would like to thank Dr Hui Gao (Huazhong University of Science and Technology) for their input.

\section{Funding}

No funding was received.

\section{Availability of data and materials}

The datasets used and/or analyzed during the present study are available from the corresponding author on reasonable request.

\section{Authors' contributions}

XJ, LY, PX, NL, CC, HW collected and analyzed the patient data. HW wrote the manuscript. All authors read and approved the final manuscript.

\section{Ethics approval and consent to participate}

Written informed consent was obtained from all patients. The present study was approved by the Ethics Committee of Sichuan University (Chengdu, China).

\section{Patient consent for publication}

All patients have provided consent for the use of their information and samples for scientific research and publication.

\section{Competing interests}

The authors declare that they have no competing interests. 


\section{References}

1. Goodarzi MO, Dumesic DA, Chazenbalk G and Azziz R: Polycystic ovary syndrome: Etiology, pathogenesis and diagnosis. Nat Rev Endocrinol 7: 219, 2011.

2. Sam S: Obesity and polycystic ovary syndrome. Obes Manag 3: 69-73, 2007.

3. Morice P, Leary A, Creutzberg C, Abu-Rustum N and Darai E: Endometrial cancer. Lancet 387: 1094-1108, 2016.

4. Bharatnur S, Kustagi P and Krishnamohan D: Endometrial carcinoma in a young woman: ' 30 is Not Immune'. J Obstet Gynaecol India 61: 686-688, 2011.

5. Pellerin GP and Finan MA: Endometrial cancer in women 45 years of age or younger: A clinicopathological analysis. Am J Obstet Gynecol 193: 1640-1644, 2005.

6. Zivanovic O, Carter J, Kauff ND and Barakat RR: A review of the challenges faced in the conservative treatment of young women with endometrial carcinoma and risk of ovarian cancer. Gynecol Oncol 115: 504-509, 2009.

7. Ding DC, Chen W, Wang JH and Lin SZ: Association between polycystic ovarian syndrome and endometrial, ovarian, and breast cancer: A population-based cohort study in Taiwan. Medicine (Baltimore) 97: e12608, 2018.

8. Park JC, Lim SY, Jang TK, Bae JG, Kim JI and Rhee JH: Endometrial histology and predictable clinical factors for endometrial disease in women with polycystic ovary syndrome. Clin Exp Reprod Med 38: 42-46, 2011.

9. Navaratnarajah R, Pillay OC and Hardiman P: Polycystic ovary syndrome and endometrial cancer. Semin Reprod Med 26: 62-71, 2008.

10. Laughlin-Tommaso SK, Hesley GK, Hopkins MR, Brandt KR, Zhu Y and Stewart EA: Clinical limitations of the international federation of gynecology and obstetrics (FIGO) classification of uterine fibroids. Int J Gynaecol Obstet 139: 143-148, 2017.

11. Rotterdam ESHRE/ASRM-Sponsored PCOS Consensus Workshop Group: Revised 2003 consensus on diagnostic criteria and long-term health risks related to polycystic ovary syndrome. Fertil Steril 81: 19-25, 2004.

12. Evans-Metcalf ER, Brooks SE, Reale FR and Baker SP: Profile of women 45 years of age and younger with endometrial cancer. Obstet Gynecol 91: 349-354, 1998.

13. Gitsch G, Hanzal E, Jensen D and Hacker NF: Endometrial cancer in premenopausal women 45 years and younger. Obstet Gynecol 85: 504-508, 1995.

14. Tran BN, Connell PP, Waggoner S, Rotmensch J and Mundt AJ: Characteristics and outcome of endometrial carcinoma patients age 45 years and younger. Am J Clin Oncol 23: 476-480, 2000.

15. Hanprasertpong J, Sakolprakraikij S and Geater A: Endometrial cancer in Thai women aged 45 years or younger. Asian $\mathrm{Pac}$ J Cancer Prev 9: 58-62, 2008.
16. Shah MM and Wright JD: Management of endometrial cancer in young women. Clin Obstet Gynecol 54: 219-225, 2011.

17. Cheung AP: Ultrasound and menstrual history in predicting endometrial hyperplasia in polycystic ovary syndrome. Obstet Gynecol 98: 325-331, 2001

18. Speert H: Carcinoma of the endometrium in young women. Surg Gynecol Obstet 88: 332-336, 1949.

19. Gottschau M, Kjaer SK, Jensen A, Munk C and Mellemkjaer L: Risk of cancer among women with polycystic ovary syndrome: A Danish cohort study. Gynecol Oncol 136: 99-103, 2015.

20. Escobedo LG, Lee NC, Peterson HB and Wingo PA: Infertility-associated endometrial cancer risk may be limited to specific subgroups of infertile women. Obstet Gynecol 77: 124-128, 1991.

21. Iatrakis G, Zervoudis S, Saviolakis A, Troulos M, Antoniou E, Sarantaki A, Lykeridou K and Kourounis G: Women younger than 50 years with endometrial cancer. Eur J Gynaecol Oncol 27: 399-400, 2006.

22. Apparao KB, Lovely LP, Gui Y, Lininger RA and Lessey BA: Elevated endometrial androgen receptor expression in women with polycystic ovarian syndrome. Biol Reprod 66: 297-304, 2002.

23. Dang CV and Semenza GL: Oncogenic alterations of metabolism. Trends Biochem Sci 24: 68-72, 1999.

24. Furberg AS and Thune I: Metabolic abnormalities (hypertension, hyperglycemia and overweight), lifestyle (high energy intake and physical inactivity) and endometrial cancer risk in a Norwegian cohort. Int J Cancer 104: 669-676, 2003.

25. Pillay OC, Te Fong LF, Crow JC, Benjamin E, Mould T, Atiomo W, Menon PA, Leonard AJ and Hardiman P: The association between polycystic ovaries and endometrial cancer. Hum Reprod 21: 924-929, 2006.

26. MacLaughlan SD, Palomino WA, Mo B, Lewis TD, Lininger RA and Lessey BA: Endometrial expression of Cyr61: A marker of estrogenic activity in normal and abnormal endometrium. Obstet Gynecol 110: 146-154, 2007.

27. Dahlgren E, Friberg LG, Johansson S, Lindström B, Odén A, Samsioe G and Janson PO: Endometrial carcinoma; ovarian dysfunction-a risk factor in young women. Eur J Obstet Gynecol Reprod Biol 41: 143-150, 1991.

28. Thiel FC and Halmen S: Low-grade endometrial stromal sarcoma-a review. Oncol Res Treat 41: 687-692, 2018.

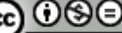

This work is licensed under a Creative Commons Attribution-NonCommercial-NoDerivatives 4.0 International (CC BY-NC-ND 4.0) License. 